\title{
Comparison of Clinicopathological Characteristics and Prognostic Factors of Gastric Cancer in Patients Aged 50 Years and Younger and in the Elderly
}

\author{
Orhan Uzun*, Aziz Serkan Senger, Selçuk Gülmez, Bülent Şen, Cem Batuhan Ofluoğlu, Ayhan \\ ÖZ, Erdal Polat and Mustafa Duman \\ University of Health Sciences Kartal Kosuyolu Higher Specialty Trainin and Research Hospital, Gastroenterology Surgery Clinic, \\ Turkey
}

*Corresponding author: Orhan UZUN, Kartal Koşuyolu High Specialty Training and Research Hospital, Denizer street, Cevizli crossroad, Cevizli/Kartal/İstanbul

\section{ARTICLE INFO \\ Received: 蔧 November 04, 2020 \\ Published: 彗 November 12, 2020}

Citation: Orhan U, Aziz Serkan S, Selçuk Gülmez, BȘ, Cem Batuhan O, et al., Comparison of Clinicopathological Characteristics and Prognostic Factors of Gastric Cancer in Patients Aged 50 Years and Younger and in Tithe Elderly. Biomed J Sci \& Tech Res 31(5)-2020. BJSTR. MS.ID.005170.

Keywords: Gastric Cancer; Young Patient; Prognostic Factors

\section{ABSTRACT}

Aim: The present study examines the clinicopathological characteristics and prognostic factors of young-age patients with gastric cancer and makes a comparison with those of older patients with gastric cancer.

Materials and Methods: The data of 262 patients who underwent curative gastrectomy at our clinic between November 2006 and December 2018 was reviewed retrospectively. A comparison was made between the 46 patients $(17.6 \%)$ aged 50 years and younger and the 216 patients (82.4\%) aged more than 50 years.

Results: Young-age gastric cancer was found to be more common in the male respondents (63\%). The Lauren diffuse histological type was more common, the number of removed lymph nodes was higher and the length of hospital stay was shorter in patients with young-age gastric cancer $(\mathrm{p}<0.05)$. The Lauren diffuse histological subtype, large tumor diameter and distal tumor localization $(\mathrm{p}<0.05)$ were identified as prognostic factors in the young patient group, whereas small tumor diameter, proximal tumor localization and the number of removed lymph nodes $(\mathrm{p}<0.05)$ were identified as prognostic factors in the elderly patient group, although there was no significant difference between the two groups in overall survival.

Conclusion: Despite the lack of a significant difference in overall survival between the young-age patients and elderly patients with gastric cancer, the prognostic factors were significantly different, including large tumor diameter, distal tumor localization and Lauren histological subtype.

\section{Introduction}

Despite a decrease in the incidence of gastric cancer, it still ranks fifth among other cancer types and it is encountered as one of the important causes of cancer-related morbidity and mortality [1]. Gastric cancer is often encountered between the ages of 50 and 70 years, although there has been an increase in the rate of gastric cancer in recent years in the young-age group, with a prevalence rate of young-age gastric cancer reported to in the range of 2-8\% [2]. Young-age gastric cancer is associated with such prognostic factors as a poorly differentiated histology and advanced disease stage $[3,4]$, Consequently, some studies have reported a poorer prognosis in young-age gastric cancer due to delays in diagnosis and the presence of poor prognostic factors [5]. Other studies, however, have reported similar or even better prognosis in young-age gastric cancer cases than in elderly patients with gastric cancer [6].

Although studies to date have identified the clinicopathological characteristics of young-age patients and the patients in the other age groups, none have addressed prognostic factors for survival in the elderly patient group, nor the effects of these prognostic 
factors in the young-age patient group. The present study identifies prognostic factors in the young-age patients and determines whether these prognostic factors have an effect in the other age group. The study similarly evaluates whether the prognostic factors in the other age group affect the young-age patients.

\section{Materials and Method}

The data of a total of 320 patients who underwent a total or subtotal gastrectomy and D2 lymph node dissection due to the presence of gastric adenocarcinoma in the Gastroenterological Surgery clinic of XXX Hospital between November 2006 and December 2015 were analyzed retrospectively. The cut-off date for the survival analysis was December 31, 2018. D2 dissections were performed in accordance with the principles of the Japanese Research Society for the Study of Gastric Cancer (JRSSG) [7]. The Tumor, Node, Metastasis (TNM) classification system of the American Joint Committee on Cancer (AJCC) (7th edn 2010) was used in the study. The data was obtained from the follow-up forms stored in our clinical database, and pathology results were recorded. Patients diagnosed with distant organ metastasis at the time of surgery (8 patients), patients with a positive peritoneal cytology (12 patients), patients who received neoadjuvant chemoradiotherapy (34 patients) and those who had positive surgical margins (4 patients) were excluded from the study, even if they underwent a gastric resection. A total of 262 patients were included in the study. Complications occurring within 30 days of surgery were defined as surgery-related complications, and were recorded.

\section{Statistical Analysis}

A Chi-square test, a Fisher's exact test, and a Mann-Whitney U test were used to analyze the differences in the study variables in the comparison of patients aged 50 years and younger and those aged older than 50 years. A Kaplan-Meier test was used to compare the survival of patients aged 50 years and younger and patients older than 50 years, and a log rank test was used to evaluate the differences between the survival curves. Univariate and multivariate analyses were performed with a stepwise Cox regression in order to identify prognostic factors for survival in patients aged 50 years and younger and in those older than 50 years. The variables included gender, Lauren histological subtype, tumor localization, tumor diameter, total number of removed lymph nodes, stage, vascular invasion, perineural invasion and presence of complications. The SPSS version 21 software package was used for the study and the level of statistical significance was set at an alpha of $<0.05$.

\section{Results}

Of the patients, $17.6 \%(n=46)$ were aged 50 years and younger and $82.4 \%(n=216)$ were older than 50 years. A comparison of clinicopathological characteristics between the two groups revealed no significant difference in terms of gender, gastrectomy type, tumor localization, $\mathrm{T}$ stage, $\mathrm{N}$ stage, TNM stage, vascular invasion, perineural invasion, tumor diameter and number of positive lymph nodes (Table 1). A significant difference was noted in the Lauren histological subtype between the two groups $(\mathrm{p}<0.05)$; and $80.4 \%$ in the young-age group and $65.3 \%$ in the elderly group had diffuse histology. The median total number of removed lymph nodes was 27 (10-73) in the young-age group and 23 (6-74) in the elderly group, showing a statistically significant difference between the two groups $(\mathrm{p}<0.05)$. The rate of postoperative complications was numerically higher in patients older than 50 years $(15.2 \%$ versus $27.8 \%$ ); and while close, the difference was not statistically significant $(\mathrm{p}=0.076)$. There was a statistically significant difference in the length of hospital stay, which was median 9 days in the youngage group and 10 days in the elderly group $(\mathrm{p}<0.05)$.

Table 1: Clinicopathological characteristics and surgical outcomes.

\begin{tabular}{|c|c|c|c|c|c|}
\hline & \multicolumn{2}{|c|}{$\leq 50$} & \multicolumn{2}{|c|}{$>\mathbf{5 0}$} & \multirow{2}{*}{$\mathbf{p}$} \\
\hline & $\mathbf{n}$ & $\%$ & $\mathbf{n}$ & $\%$ & \\
\hline \multicolumn{6}{|c|}{ Gender } \\
\hline Male & 29 & $63.00 \%$ & 150 & $69.40 \%$ & \multirow{2}{*}{0.397} \\
\hline Female & 17 & $37.00 \%$ & 66 & $30.60 \%$ & \\
\hline \multicolumn{6}{|c|}{ Gastrectomy } \\
\hline Subtotal & 27 & $58.70 \%$ & 107 & $49.50 \%$ & \multirow{2}{*}{0.259} \\
\hline Total & 19 & $41.30 \%$ & 109 & $50.50 \%$ & \\
\hline \multicolumn{6}{|c|}{ Lauren histological type } \\
\hline Intestinal & 9 & $19.60 \%$ & 75 & $34.70 \%$ & \multirow{2}{*}{$0.046^{*}$} \\
\hline Diffuse & 37 & $80.40 \%$ & 141 & $65.30 \%$ & \\
\hline \multicolumn{6}{|c|}{ Location } \\
\hline Proximal & 6 & $13.00 \%$ & 52 & $24.10 \%$ & \multirow{3}{*}{0.239} \\
\hline Middle & 13 & $28.30 \%$ & 59 & $27.30 \%$ & \\
\hline Distal & 27 & $58.70 \%$ & 105 & $48.60 \%$ & \\
\hline
\end{tabular}




\begin{tabular}{|c|c|c|c|c|c|}
\hline \multicolumn{6}{|c|}{ Depth of invasion } \\
\hline $\mathrm{T} 1$ & 4 & $8.70 \%$ & 21 & $9.70 \%$ & \multirow{4}{*}{0.577} \\
\hline $\mathrm{T} 2$ & 6 & $13.00 \%$ & 21 & $9.70 \%$ & \\
\hline $\mathrm{T} 3$ & 16 & $34.80 \%$ & 97 & $44.90 \%$ & \\
\hline $\mathrm{T} 4$ & 20 & $43.50 \%$ & 77 & $35.60 \%$ & \\
\hline \multicolumn{6}{|c|}{ Lymph node metastasis } \\
\hline N0 & 16 & $34.80 \%$ & 71 & $32.90 \%$ & \multirow{4}{*}{0.525} \\
\hline N1 & 5 & $10.90 \%$ & 43 & $19.90 \%$ & \\
\hline N2 & 8 & $17.40 \%$ & 36 & $16.70 \%$ & \\
\hline N3 & 17 & $37.00 \%$ & 66 & $30.60 \%$ & \\
\hline \multicolumn{6}{|c|}{ TNM Stage } \\
\hline $1 \mathrm{~A}$ & 4 & $8.70 \%$ & 20 & $9.30 \%$ & \multirow{7}{*}{0.947} \\
\hline $1 \mathrm{~B}$ & 2 & $4.30 \%$ & 13 & $6.00 \%$ & \\
\hline $2 \mathrm{~A}$ & 8 & $17.40 \%$ & 34 & $15.70 \%$ & \\
\hline $2 \mathrm{~B}$ & 6 & $13.00 \%$ & 28 & $13.00 \%$ & \\
\hline $3 \mathrm{~A}$ & 6 & $13.00 \%$ & 37 & $17.10 \%$ & \\
\hline 3B & 9 & $19.60 \%$ & 29 & $13.40 \%$ & \\
\hline $3 \mathrm{C}$ & 11 & $23.90 \%$ & 55 & $25.50 \%$ & \\
\hline \multicolumn{6}{|c|}{ Vascular invasion } \\
\hline Negative & 17 & $37.00 \%$ & 81 & $37.70 \%$ & \multirow{2}{*}{0.927} \\
\hline Positive & 29 & $63.00 \%$ & 134 & $62.30 \%$ & \\
\hline \multicolumn{6}{|c|}{ Perineural invasion } \\
\hline Negative & 16 & $34.80 \%$ & 69 & $32.20 \%$ & \multirow{2}{*}{0.739} \\
\hline Positive & 30 & $65.20 \%$ & 145 & $67.80 \%$ & \\
\hline \multicolumn{6}{|c|}{ Complication } \\
\hline No & 39 & $84.80 \%$ & 156 & $72.20 \%$ & \multirow{2}{*}{0.076} \\
\hline Yes & 7 & $15.20 \%$ & 60 & $27.80 \%$ & \\
\hline \multirow{5}{*}{$\begin{array}{c}\text { Tm Diameter }(\mathrm{cm}) \\
\text { Total number of lymph nodes } \\
\text { Number of positive lymph nodes } \\
\text { Length of hospital stay }\end{array}$} & Median & Min-Max & Median & Min-Max & \\
\hline & 4 & 11-Jan & 5 & $0.4-18$ & 0.442 \\
\hline & 27 & Oct-73 & 23 & Jun-74 & 0.052 \\
\hline & 4 & $0-29$ & 2 & $0-48$ & 0.37 \\
\hline & 9 & Jun-35 & 10 & $0-158$ & $0.048^{*}$ \\
\hline
\end{tabular}

Note: *p $<0.05$

No significant difference in survival was noted between the patients aged 50 years and younger and those older than 50 years $(\mathrm{p}>0.05)$, with $50 \%(\mathrm{n}=23)$ of the patients aged 50 years and younger and $53.7 \%(n=116)$ of the patients older than 50 years surviving (Figure 1), (Table 2). The prognostic factors for survival in patients aged 50 years and younger and in patients older than 50 years were analyzed using a Cox regression analysis. The Lauren

Table 2: Comparison of survival according to age using Kaplan-Meier test.

\begin{tabular}{|c|c|c|c|c|c|}
\hline \multicolumn{7}{|c|}{ Log-rank test } \\
\hline & Non-survivors & Survivors & Estimate (mean) \pm SE & $\mathbf{9 5 \% ~ C I ~}$ & p value \\
\hline$\leq 50$ & $23(50 \%)$ & $23(50 \%)$ & $73.133 \pm 7.686$ & $(58.069-88.197)$ & 0.774 \\
\hline Overall & $100(46.3 \%)$ & $116(53.7 \%)$ & $62.833 \pm 5.734$ & $(51.595-74.072)$ & \\
\hline
\end{tabular}

Note: CI: Confidence Interval 
Table 3: Univariate and multivariate analyses in patients with gastric cancer aged 50 years and younger.

\begin{tabular}{|c|c|c|c|c|}
\hline & \multicolumn{2}{|c|}{ Univarite Analysis } & \multicolumn{2}{|c|}{ Multivarite Analysis } \\
\hline & OR $(95 \% \mathrm{CI})$ & $\mathbf{p}$ & OR $(95 \% \mathrm{CI})$ & $\mathbf{p}$ \\
\hline \multicolumn{5}{|c|}{ Gender } \\
\hline Male & 1.584 & \multirow{2}{*}{0.31} & 1.636 & \multirow{2}{*}{0.534} \\
\hline Female & $(.652-3.850)$ & & $(.347-7.715)$ & \\
\hline \multicolumn{5}{|c|}{ Lauren histological type } \\
\hline Intestinal & 3.717 & \multirow{2}{*}{$0.027^{*}$} & 0.426 & \multirow{2}{*}{0.33} \\
\hline Diffuse & $(1.159-11.917)$ & & $(.076-2.372)$ & \\
\hline \multicolumn{5}{|c|}{ Location } \\
\hline Upper & $\begin{array}{c}0.922 \\
(.255-3.340)\end{array}$ & $0.025^{*}$ & $\begin{array}{c}11.706 \\
1.129-121.413\end{array}$ & $0.017^{*}$ \\
\hline Middle & $\begin{array}{c}5.44 \\
(1.561-18.958)\end{array}$ & 0.902 & $\begin{array}{c}1.09 \\
(.163-7.310)\end{array}$ & $0.039 *$ \\
\hline Lower & & $0.008^{*}$ & & 0.929 \\
\hline \multicolumn{5}{|c|}{ Tumor diameter } \\
\hline$\leq 4 \mathrm{~cm}$ & $\begin{array}{c}3.913 \\
(1.276-12.002)\end{array}$ & $0.017^{*}$ & $\begin{array}{c}6.855 \\
(1.097-42.813)\end{array}$ & $0.039^{*}$ \\
\hline$>4 \mathrm{~cm}$ & $\begin{array}{c}1.037 \\
1.037(1.003-1.071) \\
\end{array}$ & $0.033^{*}$ & $\begin{array}{c}1.054 \\
(1.002-1.109) \\
\end{array}$ & $0.041^{*}$ \\
\hline \multicolumn{5}{|c|}{ Total number of lymph nodes Stage } \\
\hline I & $\begin{array}{c}0.61 \\
(.196-1.900) \\
\end{array}$ & 0.465 & $\begin{array}{c}269 \\
(.042-1.735) \\
\end{array}$ & 0.287 \\
\hline II & $\begin{array}{c}1.138 \\
(.377-3.435) \\
\end{array}$ & 0.394 & $\begin{array}{c}0.439 \\
(.025-7.680) \\
\end{array}$ & 0.168 \\
\hline III & & 0.819 & & 0.573 \\
\hline Vascular invasion & $\begin{array}{c}1.342 \\
.564-3.191 \\
\end{array}$ & 0.506 & $\begin{array}{c}0.764 \\
(.184-3.177)\end{array}$ & 0.711 \\
\hline Perineural invasion & $\begin{array}{c}1.302 \\
(.529-3.202)\end{array}$ & 0.566 & $\begin{array}{c}0.634 \\
(.126-3.199)\end{array}$ & 0.581 \\
\hline Complication & $\begin{array}{c}0.21 \\
(.037-1.182)\end{array}$ & 0.077 & $\begin{array}{c}0.385 \\
(.038-3.843)\end{array}$ & 0.416 \\
\hline
\end{tabular}

Note: OR: Ods Ratio, CI: Confidence Interval, * $\mathrm{p}<0.05$

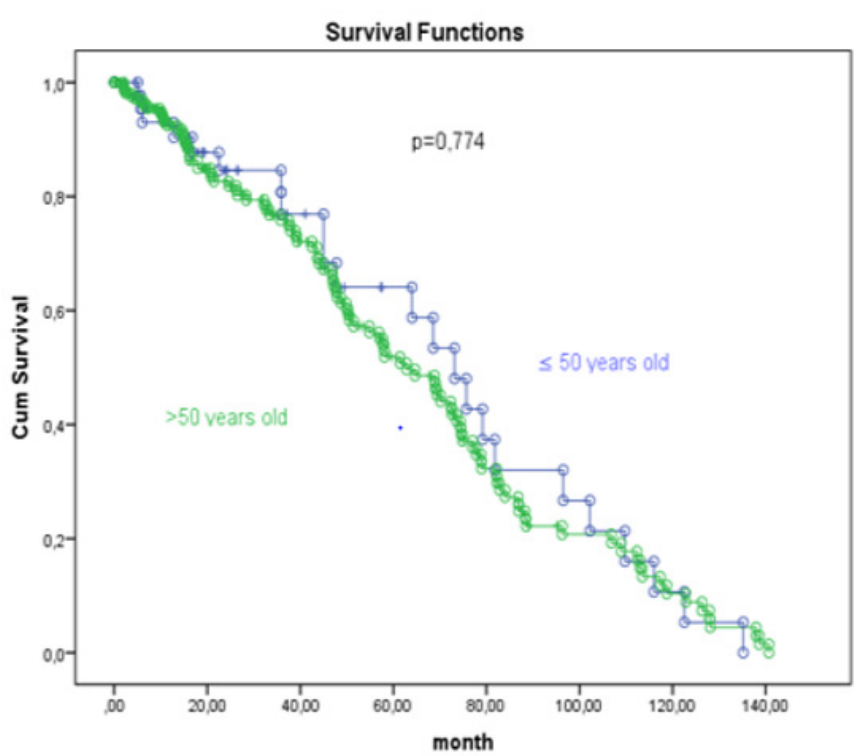

Figure 1: Overall survival comparison of age-related. 
In a univariate analysis of the patients aged older than 50 years, proximal tumor localization, total number of removed lymph nodes and tumor diameter smaller than $4 \mathrm{~cm}$ were identified as prognostic factors affecting the risk of mortality. Tumor diameter smaller than $4 \mathrm{~cm}$ and total number of removed lymph nodes were identified as prognostic factors affecting the risk of mortality in a multivariate analysis (Table 4). The prognostic factors in patients aged 50 years and younger were partially different to those in patients older than 50 years. Although the total number of removed lymph nodes was identified as a prognostic factor in both groups in a stepwise Cox regression analysis, distal tumor localization and large tumor diameter were significant prognostic factors in the young-age group, whereas proximal tumor localization and small tumor diameter were significant in the elderly patients. The Lauren diffuse histological subtype was found to be significant in a univariate analysis of the young-age patient group, but insignificant in the elderly patient group.

Table 4: Univariate and multivariate analyses in patients with gastric cancer aged older than 50 years.

\begin{tabular}{|c|c|c|c|c|}
\hline & \multicolumn{2}{|c|}{ Univarite Analysis } & \multicolumn{2}{|c|}{ Multivarite Analysis } \\
\hline & OR $(95.0 \% \mathrm{CI})$ & $\mathbf{p}$ & OR $(95.0 \% \mathrm{CI})$ & $\mathbf{p}$ \\
\hline \multicolumn{5}{|c|}{ Gender } \\
\hline Male & 1.002 & \multirow{2}{*}{0.991} & 0.988 & \multirow{2}{*}{0.966} \\
\hline Female & $(.660-1.522)$ & & $(.570-1.712)$ & \\
\hline \multicolumn{5}{|c|}{ Lauren histological type } \\
\hline Intestinal & 0.995 & 0.979 & 1.034 & 0.891 \\
\hline Diffuse & $(.659-1.502)$ & $0.037^{*}$ & $(.641-1.668)$ & 0.305 \\
\hline \multicolumn{5}{|c|}{ Location } \\
\hline & 0.99 & & 0.776 & \\
\hline Opper & $(.573-1.710)$ & 0.971 & $(.386-1.563)$ & 0.478 \\
\hline Intive & 0.591 & $0.028^{*}$ & 0.646 & 0.128 \\
\hline Lower & $(.369-.945)$ & & $(.368-1.135)$ & \\
\hline \multicolumn{5}{|c|}{ Tumor diameter } \\
\hline$\leq 4 \mathrm{~cm}$ & $0.605(.403-.908)$ & $0.015^{*}$ & $0.538(.341-.848)$ & $0.008^{*}$ \\
\hline$>4 \mathrm{~cm}$ & $1.028(1.011-1.046)$ & $0.001^{*}$ & $1.022(1.002-1.042)$ & $0.030^{*}$ \\
\hline \multicolumn{5}{|c|}{ Total number of lymph nodes Stage } \\
\hline I & \multirow{4}{*}{$\begin{array}{c}1.285 \\
(.783-2.108) \\
1.156 \\
(.687-1.942)\end{array}$} & 0.611 & \multirow{4}{*}{$\begin{array}{c}1.742 \\
(.953-3.183) \\
1.555 \\
(.737-3.278) \\
\end{array}$} & 0.196 \\
\hline 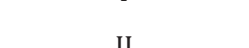 & & 0.021 & & 0071 \\
\hline 11 & & 0.321 & & 0.071 \\
\hline III & & 0.585 & & 0.246 \\
\hline \multirow{6}{*}{$\begin{array}{c}\text { Vascular invasion } \\
\text { Perineural invasion } \\
\text { Complication }\end{array}$} & \multirow{6}{*}{$\begin{array}{l}(.739-1.656) \\
0.841 \\
(.563-1.258)\end{array}$} & & 0.828 & \multirow{6}{*}{$\begin{array}{l}0.486 \\
0.109 \\
0.409\end{array}$} \\
\hline & & & $(.486-1.409)$ & \\
\hline & & 0.623 & 0.648 & \\
\hline & & 0.399 & $(.382-1.102)$ & \\
\hline & & 0.128 & 0.814 & \\
\hline & & & $(.500-1.326)$ & \\
\hline
\end{tabular}

Note: OR: Ods Ratio, CI: Confidence Interval, ${ }^{*} \mathrm{p}<0.05$

\section{Discussion}

Gastric cancer is often more common in the 50-70 years age group, with a higher prevalence in males than in females in this age group, with a male-to-female ratio of 2:1 [8]. Gastric cancer is less prevalent in the young-age group, with a reported prevalence of $2-9 \%$ in literature [9]. Although various studies have reported different results, young-age gastric cancer is associated with poor prognosis; the incidence is higher in females; and the disease often occurs in the proximal section of the stomach and manifests in the advanced stage with a Lauren diffuse histology, and particularly with a signet ring histology [10]. Sex hormones or analogues have been identified as occurring more often in females [11]. Although the present study found no difference between the two groups in terms of prognosis, there was a male predominance in all age groups and in the young patients with gastric cancer.

Furthermore, the number of removed lymph nodes was significantly lower and the length of hospital stay was significantly shorter in young patients with gastric cancer. The rate of 
encountering a Lauren diffuse-type histology in gastric cancer is around $30 \%$ and is more frequent in young-age gastric cancer cases and in females [12]. In a Lauren diffuse-type histology, cells appear scattered in the stroma or as a cluster of cells rather than having tubular or glandular appearance due to loss of cellular adhesion [13]. Gastric cancers with a Lauren diffuse-type histology are associated with poorer prognosis due to the more aggressive tumor behavior with advanced T stage and N stage [14]. In our study, the patients with a Lauren diffuse-type histology were mostly in the young-age patient group, and the Lauren diffuse histology was identified as a prognostic factor in a univariate analysis, but not in a multivariate analysis.

The rate of complications related to surgery was lower and the length of hospital stay was shorter in young patients who were operated on for gastric cancer. The present study produced similar results. Large tumor size is one of the poor prognostic factors in gastric cancer (Zhou et al. 2019). The likelihood of lymph node involvement and peritoneal involvement increases with increasing tumor size, and patients with gastric cancer present with early recurrence, and for this reason, more aggressive treatment options are required as the tumor size increases [15]. The 5-year survival rate of patients with a tumor diameter less than $5 \mathrm{~cm}$ is $45.5 \%$, whereas 5 -year survival is $24.8 \%$ in patients with a tumor diameter greater than $5 \mathrm{~cm}$ [16]. A lower cut-off level was used for the tumor diameter in the present study, with a tumor diameter greater than $4 \mathrm{~cm}$ identified as a prognostic factor in young patients with gastric cancer. Different from other studies, tumor diameter smaller than $4 \mathrm{~cm}$ was identified as a prognostic factor in patients with gastric cancer who were aged older than 50 years.

There has been an increase in the prevalence of proximal gastric cancer and a decrease in the prevalence of distal gastric cancer since the 1970s, and epidemiological studies suggest different etiologies and biological behaviors for these two tumor localizations [17]. Proximal gastric cancer is associated with a poorer prognosis due to the more frequent occurrence in the elderly patients with advanced stage and a poor histological subtype [18]. Aside from tumor localization, the location of the tumor within the stomach is another prognostic factor, in that tumors located in the greater curvature are associated with poorer prognosis than those located in the lesser curvature [19]. Young-age gastric cancer is often located in the middle and distal stomach, though the prognostic value of this localization is controversial [20]. In the present study, young-age gastric cancers were mostly localized to the middledistal stomach, the localization being of no prognostic value.

Proximal gastric cancer was more common in the elderly patients, and the localization had a prognostic value. National Comprehensive Cancer Network (NCCN) guidelines state that a minimum 15 lymph nodes must be removed in order to accurately determine the number of positive lymph nodes [21]. The status of the lymph nodes is related entirely to prognosis and recurrence, and the removal of 22 or more lymph nodes increases overall survival, particular in patients with advanced $\mathrm{T}$ stage $[22,23]$. The number of removed lymph nodes was also found to have an effect on prognosis in the present study.

\section{Conclusion}

young patients with gastric cancer have different clinicopathological characteristics to elderly patients with gastric cancer. Furthermore, different to elderly patients, a Lauren diffusetype histology and a large tumor diameter appear to be significant prognostic factors in young-age patients with gastric cancer. The Lauren histological subtype is of no prognostic significance in elderly patients with gastric cancer, in whom small tumor diameter appears to be an important prognostic factor.

\section{References}

1. Ferlay J, M Colombet, I Soerjomataram, C Mathers, DM Parkin, et al. (2019) "Estimating the Global Cancer Incidence and Mortality in 2018: GLOBOCAN Sources and Methods." International Journal of Cancer 144(8): 1941-1953.

2. Rona Kais A, Katrin Schwameis, Joerg Zehetner, Kamran Samakar, Kyle Green, et al. (2017) "Gastric Cancer in the Young: An Advanced Disease with Poor Prognostic Features." Journal of Surgical Oncology 115(4): 371-75.

3. Takatsu, Yukiko, Naoki Hiki, Souya Nunobe (2015) "Clinicopathological Features of Gastric Cancer in Young Patients." Gastric Cancer volume 19: 472-478.

4. Saito Hiroaki, Seigo Takaya, Yoji Fukumoto, Tomohiro Osaki, Shigeru Tatebe, et al. (2012) Clinicopathologic Characteristics and Prognosis of Gastric Cancer in Young Patients.

5. Suh Do Dam, Seong Tae Oh, Jeong Hwan Yook, Byung-sik Kim (2017) "Differences in the Prognosis of Early Gastric Cancer According to Sex and Age. Therap Adv Gastroenterol 10(2): 219-229.

6. Liu Shushang, Fan Feng, Guanghui Xu, Zhen Liu, Yangzi Tian, et al. (2016) "Clinicopathological Features and Prognosis of Gastric Cancer in Young Patients." BMC Cancer pp. 1-7.

7. Sano Takeshi, Yasuhiro Kodera (2011) "Japanese Classification of Gastric Carcinoma: 3rd English Edition." Gastric Cancer 14(2): 101-112.

8. Chung Hye Won, Sung Hoon Noh, Jong baeck Lim (2010) "Analysis of Demographic Characteristics in 3242 Young Age Gastric Cancer Patients in Korea" World J Gastroenterol 16(2): 256-263.

9. Nakamura Rieko, Yoshiro Saikawa, Tsunehiro Takahashi, Hiroya Takeuchi (2011) "Retrospective Analysis of Prognostic Outcome of Gastric Cancer in Young Patients. Int J Clin Oncol 16(4): 328-334.

10. Kulig Jan, Tadeusz Popiela, Piotr Kolodziejczyk, Marek Sierzega, Joanna Jedrys (2008) "Clinicopathological Profile and Long-Term Outcome in Young Adults with Gastric Cancer: Multicenter Evaluation of 214 Patients." Multicenter Study 393(1): 37-43.

11. Hsieh Feng Jen, Yu Chao Wang, Jun Te Hsu, Keng Hao Liu, Chun Nan Yeh, et al. (2012) "Clinicopathological Features and Prognostic Factors of Gastric Cancer Patients Aged 40 Years or Younger." Journal of Surgical Oncology 105(3): 304-309.

12. Li, Xiayi, Xueru Zhu, Yiwei Wang, Ruifen Wang, Lifeng Wang, et al. (2019) "Prognostic Value and Association of Lauren Classification with VEGF and VEGFR-2 Expression in Gastric Cancer." Oncology Letters 18(5): 4891-4899. 
13. Stiekema JA, Cats A Kuijpers, F Van Coevorden, H Boot, EPM Jansen (2013) "Surgical Treatment Results of Intestinal and Diffuse Type Gastric Cancer. Implications for a Differentiated Therapeutic Approach ?" European Journal of Surgical Oncology 39(7): 686-693.

14. Qiu Miao zhen, Mu yan Cai, Dong sheng Zhang, Zhi qiang Wang, De shen Wang, et al. (2013) Clinicopathological Characteristics and Prognostic Analysis of Lauren Classification in Gastric Adenocarcinoma in China.

15. Zhou Liyuan, Weihua Li, Shaoxin Cai, Changshun Yang (2019) "Large Tumor Size Is a Poor Prognostic Factor of Gastric Cancer with Signet Ring Cell: Results from the Surveillance, Epidemiology, and End Results Database." Medicine 98(40): e17367.

16. Silva Edaise M, Maria D Begnami, José Humberto, TG Fregnani, Adriane G. et al. (2008) "Cadherin-Catenin Adhesion System and Mucin Expression: A Comparison between Young and Older Patients with Gastric Carcinoma." Gastric Cancer 11(3): 149-159.

17. Wang Xiang, Fangfang Liu, Yumin Li, Song Tang, Yingtai Chen, et al. (2019) “Comparison on Clinicopathological Features, Treatments and Prognosis between Proximal Gastric Cancer and Distal Gastric Cancer: A National Cancer Data Base Analysis." Journal of Cancer 10(14): 31453153.

18. Pacelli F, V Papa, P Caprino (2001) "Proximal Compared with Distal Gastric Cancer: Multivariate Analysis of Prognostic Factors." The American Surgeon 67(7): 697-703.

\section{ISSN: 2574-1241}

DOI: 10.26717/BJSTR.2020.31.005170

Orhan Uzun. Biomed J Sci \& Tech Res

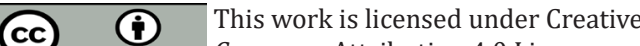
Commons Attribution 4.0 License

Submission Link: https://biomedres.us/submit-manuscript.php
19. Jung Yoon Ju, Ho Seok Seo, Ji Hyun Kim, Cho Hyun Park, Han Hong Lee (2017) "Cross-Sectional Location of Gastric Cancer Affects the Long-Term Survival of Patients as Tumor Invasion Deepens." Annals of Surgical Oncology 24(13): 3947-3953.

20. Kim Dong yi, Jae kyoon Joo, Seong yeob Ryu, Young kyu Park, Young jin Kim, et al. (2005) "Clinicopathologic Characteristics of Gastric Carcinoma in Elderly Patients: A Comparison with Young Patients." World J Gastroenterol 11(1): 22-26.

21. In Haejin, I Solsky, B Palis, M Langdon Embry, J Ajani, et al. (2017) "Validation of the $8^{\text {th }}$ Edition of the AJCC TNM Staging System for Gastric Cancer Using the National Cancer Database." Annals of Surgical Oncology 24(12): 3683-3691.

22. Ji Xin, Zhao De Bu, Zi Yu Li, Ai Wen Wu, Lian Hai Zhang, et al. (2017) Prognostic Significance of the Total Number of Harvested Lymph Nodes for Lymph Node-Negative Gastric Cancer Patients.

23. Tsutsuyama, Masayuki, Mitsuro Kanda, Seiji Ito, Yoshinari Mochizuki (2019) "Tumor Size $\geq 50 \mathrm{Mm}$ as an Independent Prognostic Factor for Patients with Stage II or III Gastric Cancer After Postoperative S-1 Monotherapy: Analysis of a Multi-Institution Dataset." World Journal of Surgery 44(1): 194-201.

BIOMEDICAL
RESEARCHES $\quad \begin{aligned} & \text { Assets of Publishing with us } \\ & \text { - Global archiving of articles }\end{aligned}$

